Research Article

\title{
Evaluating the Potential of Laser Beam Quality Improvement by Adaptive Optics System
}

\author{
Kai Han, ${ }^{1,2}$ Wenda Cui $\mathbb{D}^{1,3}$ Yi Yang, ${ }^{1,3}$ Fengjie Xi, ${ }^{1,3}$ Xiao Li, ${ }^{1,3}$ and Shaojun $\mathrm{Du}^{1,3}$ \\ ${ }^{1}$ College of Advanced Interdisciplinary Studies, National University of Defense Technology, Changsha 410073, China \\ ${ }^{2}$ State Key Laboratory of Pulsed Power Laser Technology, Changsha 410073, China \\ ${ }^{3}$ Hunan Provincial Key Laboratory of High Energy Laser Technology, Changsha 410073, China
}

Correspondence should be addressed to Wenda Cui; cui_wenda@126.com

Received 13 August 2019; Accepted 18 November 2019; Published 5 December 2019

Academic Editor: E. Bernabeu

Copyright (c) 2019 Kai Han et al. This is an open access article distributed under the Creative Commons Attribution License, which permits unrestricted use, distribution, and reproduction in any medium, provided the original work is properly cited.

AO (adaptive optics) systems have been extensively used to improve the beam quality in high-energy lasers; however, few studies have focused on how much the beam quality could be improved. A novel evaluation criteria and factor is presented in this paper. The factor, defined as power in ring (PIR), is expressed by distinguishing the low- and high-spatial frequency components in the far-field laser intensity distribution. Beams with different PIR values are generated in our model, and then they are compensated by AO systems. Calculation result shows that the PIR factor could evaluate a laser beam's improvement potential by an AO system quantitatively.

\section{Introduction}

Beam quality of the light exiting from lasers is usually quite poor in high-energy lasers) because of multimode oscillation, thermal effect, phase perturbation, and so on [1-4]. Nowadays, AO (adaptive optics) systems have been extensively used to improve the beam quality in high-energy lasers [5-9]. By compensating the phase aberration, AO systems could make the far-field intensity distribution very close to the ideal. Namely, beam quality of lasers could be improved apparently by $\mathrm{AO}$ systems.

Various beam quality evaluation factors have been presented so far, such as the PIB (power in bucket), $M^{2}$, and $\beta$ factors [10-14]. Lots of researchers have studied the phase aberration effects on the laser beam quality. Based on these evaluation factors, the investigations have showed the intensity perturbation in long-distance propagation and the compensation effect by the AO system, as well as the relationship between beam quality and different Zernike polynomials [15-20]. All these investigations have revealed that beam quality evaluation factors are closely correlated with phase aberrations and the $\mathrm{AO}$ compensation ability. However, none of the factors used in those studies could directly evaluate how much the beam quality would be improved by AO systems. Since the far-field light intensity distribution is tightly related to the phase spatial characteristics, according to the angular spectrum theory, a corresponding evaluation factor named power in ring based on intensity analysis could be defined.

In this paper, we first analyzed the long-propagation light intensity distribution with different Zernike phase aberrations. Then, we presented a new factor, which could evaluate how much the beam will be improved by $\mathrm{AO}$ systems. Different from the classic encircled energy, PIB, $M^{2}$, and $\beta$ factors, this factor exacts certain spatial frequency components in the far-field light intensity distribution by detecting power in some diffraction radiuses. Since an AO system's compensation ability is obviously dependent on lasers' spatial frequency $[21,22]$, the improvement potential by an $\mathrm{AO}$ system could be evaluated straightforwardly by this factor. In the end, we simulated the $\mathrm{AO}$ compensation effects with different new factors and $\mathrm{AO}$ systems.

\section{Factor Definition}

Typical beam quality factors like PIB (power in bucket), $M^{2}$, and $\beta$ factors are usually obtained just by light intensity 
detection, which is reliable and easy to operate. So, the farfield light intensity distribution was calculated to present a new evaluation factor in this section. Since AO compensation efficiency is closely relevant to spatial frequency of phase distributions, phase aberrations with lower and higher spatial frequency components are generated, respectively, in our model, which are reconstructed by a random linear combination of different Zernike polynomials. Output lasers with poor beam quality are simulated by adding the phase aberrations to Gauss beams, and intensity distributions are obtained based on the angular spectrum transmission method as shown in Figure 1. The common $\beta$ factor is used in the model to estimate beam qualities of different beams, which is defined as the ratio of the actual beam divergence angle to the ideal one.

In Figure 1(a), no Zernike phase is added, so only ideal diffraction rings exist around the central spot. In Figures 1(b) and 1(c), the far-field intensity distributions were calculated with the $1^{\text {st }}$ to $14^{\text {th }}$ or $91^{\text {st }}$ to $104^{\text {th }}$ order Zernike aberrations. The $91^{\text {st }}$ to $104^{\text {th }}$ order Zernike aberrations would introduce more higher spatial frequency components than the $1^{\text {st }}$ to $14^{\text {th }}$ order Zernike aberrations. In Figure 1(b), the $1^{\text {st }}$ to $14^{\text {th }}$ Zernike polynomials are added to the output laser beam, so some irregular laser speckles have emerged around the central diffraction spot. Similarly, the $91^{\text {st }}$ to $104^{\text {th }}$ Zernike polynomials are added as shown in Figure 1(c), some speckles appear around the central diffraction spot either, but they are quite weak and intensity distribution is similar with Figure 1(a), so it seems easy to be rectified by an AO system compared with the beam in Figure 1(b). However, the beam in Figure 1(b) is actually easier to be compensated because it is added with lower-order Zernike phases. Since $\beta$ factors are both 4.5 in Figures 1(b) and 1(c) by adjusting the amplitudes of Zernike coefficients, which means the $\beta$ factor cannot distinguish the improvement potential of beams with phase aberrations containing different spatial frequency components.

To present a new evaluation factor, we need to analyze the spatial frequency characteristics of far-field intensity. According to the angular spectrum theory, higher spatial frequency beam propagates with a larger diffraction angle, so it corresponds to power in the outer ring of the intensity distribution. Under the same conditions shown in Figure 1, power in different diffraction rings is calculated as shown in Figure 2.

As shown in Figure 2, the horizontal axis $n$ indicates the $n$-th diffraction ring and the vertical axis is the power in the ring. Comparing Figures 2(a) 2(c), power distribution in diffraction rings is apparently different with distortion phases of different order Zernike polynomials. It is apparent that power percentage in the first diffraction radius ring, namely, the central spot is the highest. In Figure 2(a), more than $83 \%$ power is concentrated in the first ring. Furthermore, as the Zernike phase is added, namely, the laser beam is modulated by the higher spatial frequency phase, fraction of power in larger rings increases. In Figure 2(b), more than $67 \%$ of power is distributed in rings larger than the first one. However, as the added Zernike order becomes higher in Figure 2(b), power in the 2nd to 4 th rings decreases as shown in Figure 2(c).
Because beam with the lower-order Zernike phase is easier to be rectified by an AO system, beam with the pattern 2 has more potential to be improved. So, a new evaluation factor named PIR (power in ring) can be defined as follows:

$$
\mathrm{PIR}=\frac{1}{0.7024} \cdot \frac{P_{\mathrm{LF}}}{P_{\mathrm{LF}}+P_{\mathrm{HF}}},
$$

where 0.7024 is the normalization coefficient representing the ratio of power in the $2 \sim 4^{\text {th }}$ ring in ideal situation to power in larger than the $4^{\text {th }}$ ring. $P_{\mathrm{LF}}$ and $P_{\mathrm{HF}}$ are the lowfrequency and high-frequency power calculated by

$$
\begin{aligned}
& P_{\mathrm{LF}}=\int_{0}^{2 \pi} \int_{R_{1}}^{R_{2}} I_{\mathrm{F}}(r, \varphi) r \mathrm{~d} r \mathrm{~d} \varphi, \\
& P_{\mathrm{HF}}=\int_{0}^{2 \pi} \int_{R_{2}}^{\infty} I_{\mathrm{F}}(r, \varphi) r \mathrm{~d} r \mathrm{~d} \varphi,
\end{aligned}
$$

in which $I_{\mathrm{F}}(r, \varphi)$ is the far-field intensity distribution in polar coordinates, $R_{1}$ is radius of the first diffraction ring, and $R_{2}$ is the $4^{\text {th }}$ diffraction ring radius:

$$
\begin{aligned}
& R_{1}=1.22 \frac{\lambda}{D} f, \\
& R_{2}=4.24 \frac{\lambda}{D} f .
\end{aligned}
$$

In our definition, power in the first ring can be considered as the zero-frequency component, which does not need rectification by an AO system, so this part can be ignored. $D$ is the effective beam diameter, which is expressed by

$$
D=2 \sqrt{2 \sigma^{2}}
$$

where $\sigma$ is the second moment of the light intensity distribution:

$$
\sigma^{2}=\frac{\iint r^{2} \cdot I_{N}(r, \varphi) r \mathrm{~d} r \mathrm{~d} \varphi}{\iint I_{N}(r, \varphi) r \mathrm{~d} r \mathrm{~d} \varphi,}
$$

in which $I_{N}$ is the near-field intensity distribution in polar coordinates.

Using equations (1) (7), PIR of any kind of laser could be obtained. According to the definition, the PIR value ranges between 0 and 1 , which indicates the low-frequency aberration percentage of the whole-phase aberration components. If the PIR value is closer to 1 , lower spatial frequency components are primary in the beam. It will be easier to be rectified by AO systems, namely, the beam has more potential to be improved. Meanwhile, only light intensity is needed in the calculation, which is convenient to be detected and does not affect the other beam quality detection equipment, so it could be applied in extensive situations easily.

\section{PIR Application}

We set up a simulation model of the AO compensation to verify the ability of the PIR factor in evaluating a laser beam's improvement potential. Beams with the same initial $\beta_{0}=4.5$ are generated by the same way in Section 2. Meanwhile, their PIR factors are calculated before compensation by the $\mathrm{AO}$ 


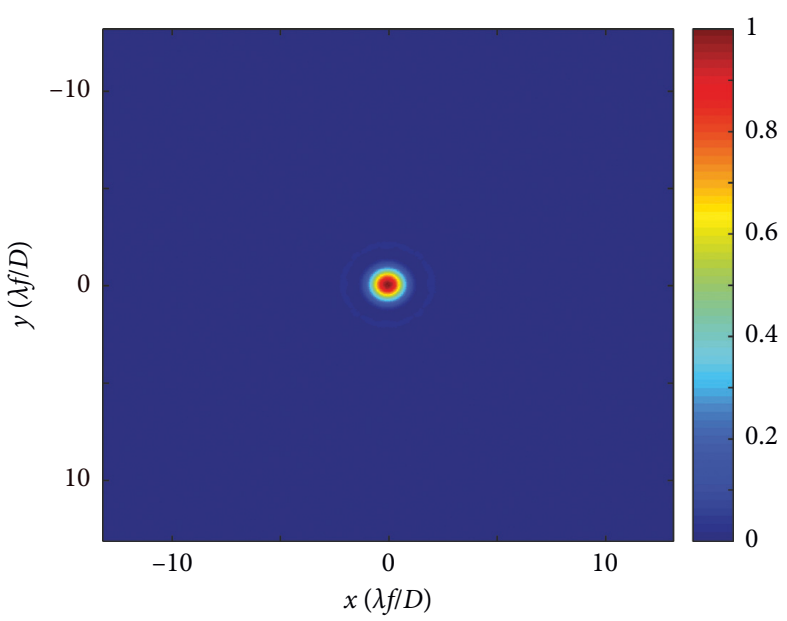

(a)

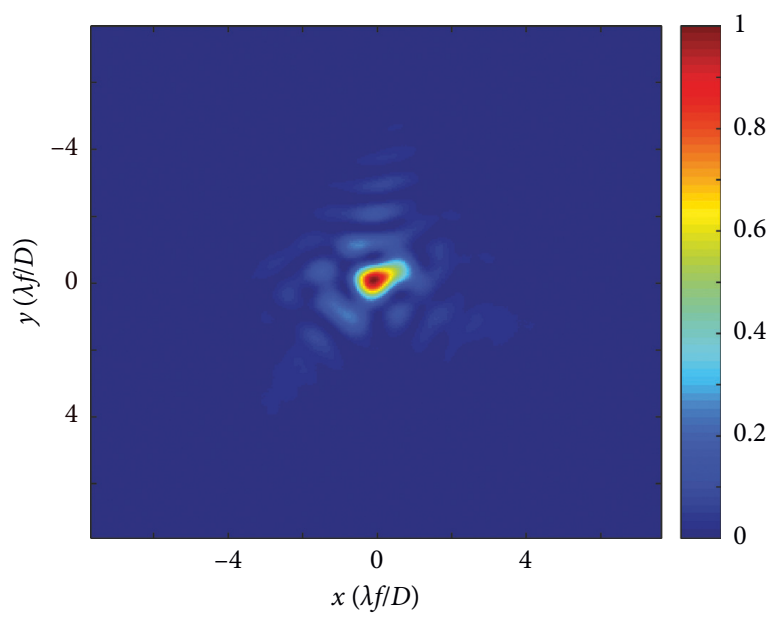

(b)

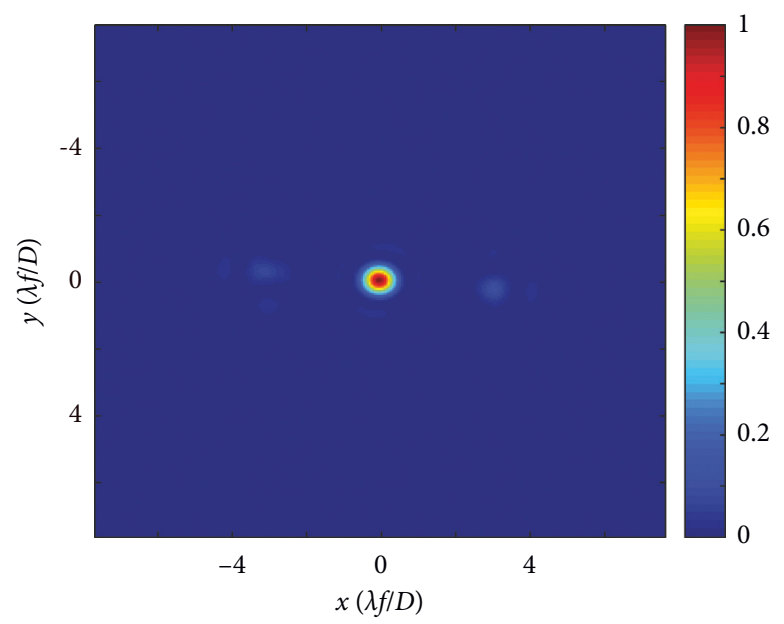

(c)

FIGURE 1: Intensity distribution with different Zernike combinations: (a) ideal beam pattern without phase aberrations, (b) beam pattern 1 with the $1^{\text {st }}$ to $14^{\text {th }}$ order Zernike phase, and (c) beam pattern 2 with the $91^{\text {st }}$ to $104^{\text {th }}$ order Zernike phase.

system based on the definition. Schematic diagram of numerical model is depicted in Figure 3.

As shown in Figure 3, beams with the same $\beta_{0}=4.5$ are transmitted into the AO system. Various DMs (deformable mirrors) with different numbers of actuators are used in the model. In the simulation, we did not consider the temporal dynamics and assumed that actuator's spatial resolution is the main limit to the AO system's compensation ability; other factors, like responsive bandwidth, rectification error, reconstruction algorithm, and control law, are considered as ideal in this model. In other words, the AO system could detect the phase distributions perfectly and control the distortion of every DM segment to form the optimal compensation phase. In Figure 3, red dots indicate the actuators, which are hexagon distributed, and beam diameter consists well with the DM. The beams are rectified by AO systems with DMs of 19, 37, 61, 91, 127, and 169 actuators. After the compensation, $\beta$ factors are calculated again and simulation results are presented in Figure 4.

As shown in Figure 4, the horizontal axis indicates lasers' PIR factors and the vertical axis is the $\beta$ factor of each beam after AO system's compensation. Since spatial frequency components of these beams' phase aberration are different, their PIR values would change a lot correspondingly. In all graphs Figures 4(a) 4(f), 176 beams with different phase aberrations are simulated and statistical results show that $\beta$ values decrease closely to 1 while these beams' PIR factors are larger than 0.9 , which means their divergence characteristics or beam qualities are improved apparently. In Figures $4(\mathrm{a}) \sim 4(\mathrm{~d})$, the $\beta$ values differ little from 4.5 when PIR is close to 0 . If PIR of a beam is close to 0 , it contains few lowspatial frequency components according to the definition in Section 2, which means it could not be improved well by AO systems.

As the amount of DM actuators increases (Figures 4(b) 4(f)), beams are improved apparently by AO system although their PIR values are lower. In Figure 4(b), the value is about 0.8, and in Figure 4(f), the value decreases to 0.3. That means as the DM actuator number increases, it is able to rectify the phase aberrations with higher spatial frequency, so even a beam with less low-spatial frequency components could be improved efficiently. 


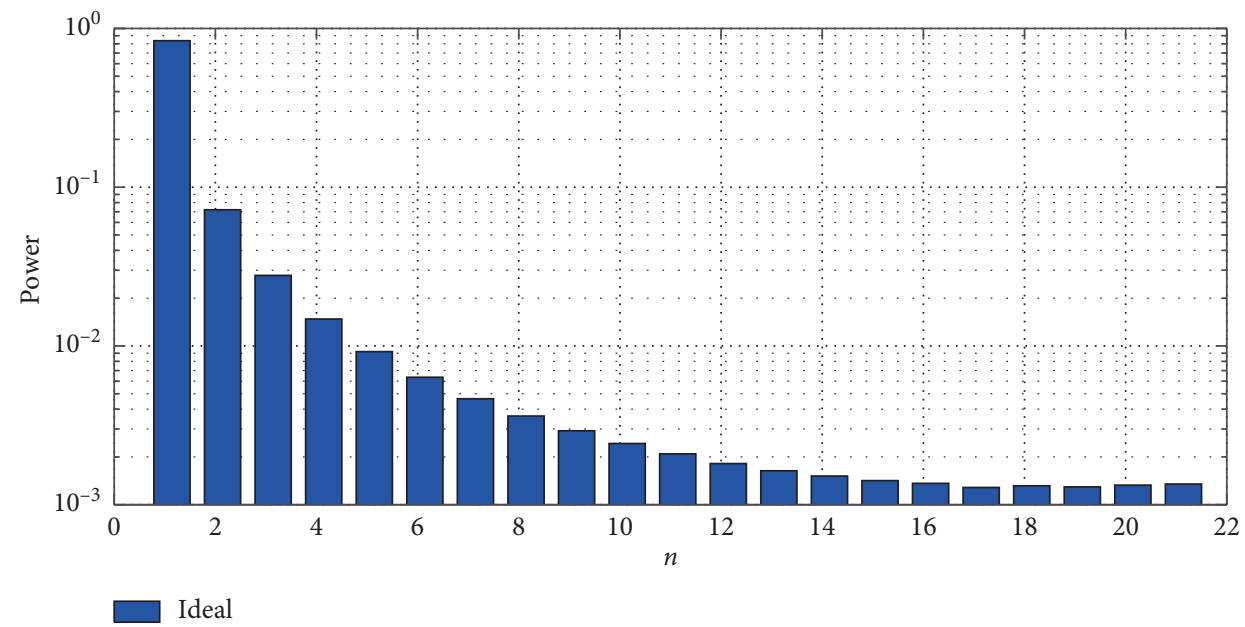

(a)

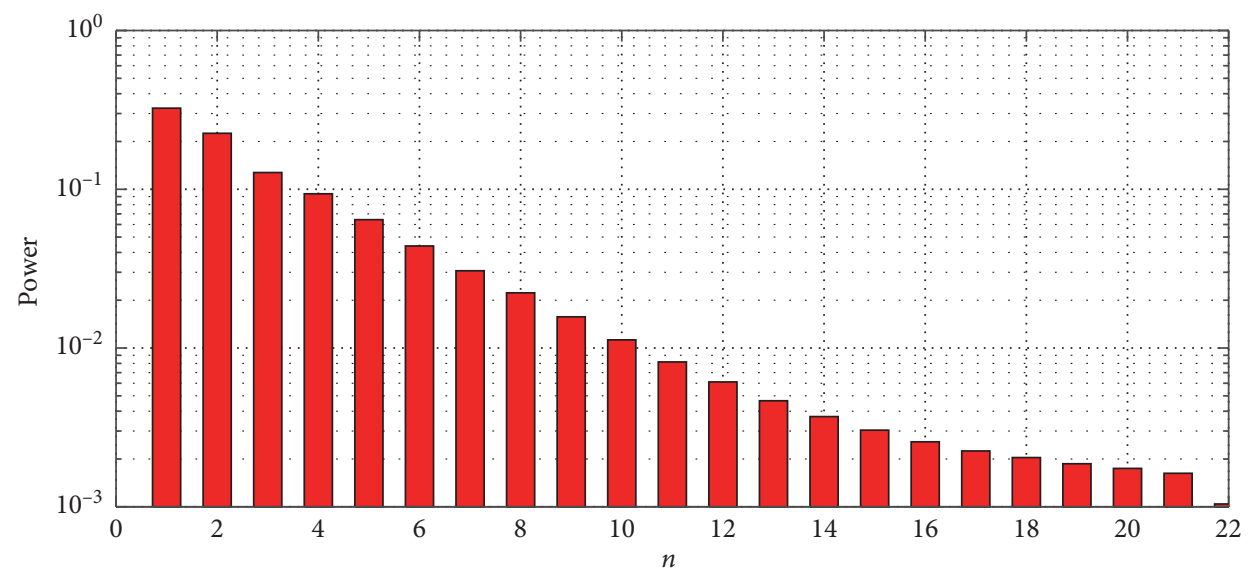

(b)

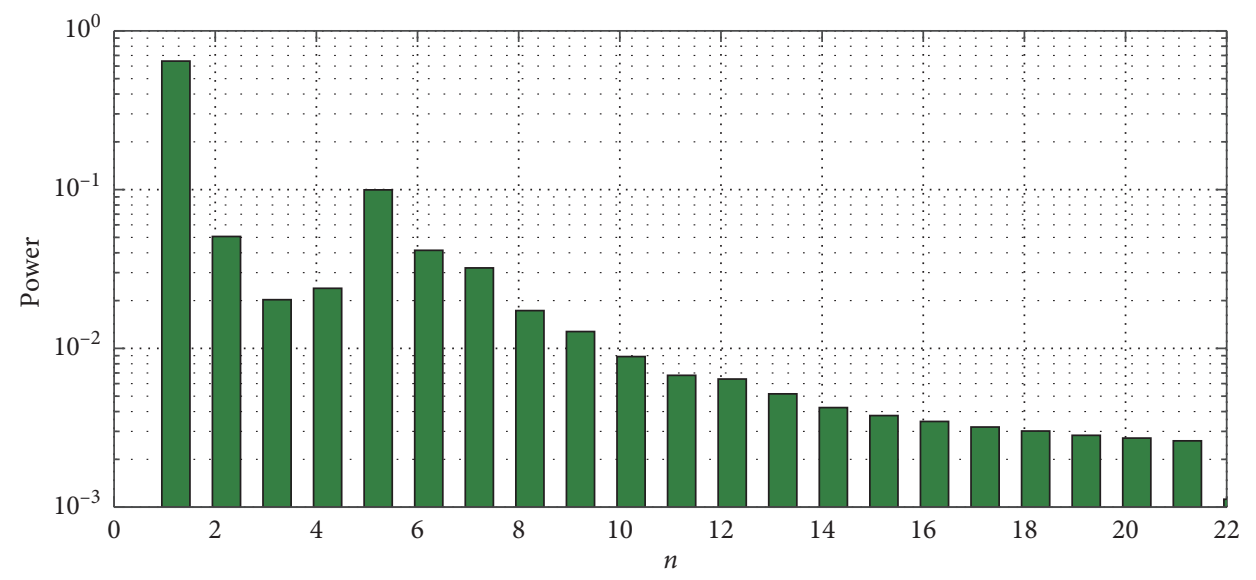

Beam pattern 2

(c)

Figure 2: Power in different diffraction radius rings with different Zernike combinations: (a) no phase aberrations, (b) $1^{\text {st }}$ to $14^{\text {th }}$ order Zernike phase, and (c) $91^{\text {st }}$ to $104^{\text {th }}$ order Zernike phase.

$\beta$ values of beams with PIR less than 0.4 decrease apparently (Figures 4(e) and 4(f)), which means they are improved well. In Figure 4(f), $\beta$ values of beams with PIR around 0.3 decrease directly to less than 1.5 after the AO compensation. The reason is that as the DM actuator number of the AO system increases to 169 , it is able to rectify 


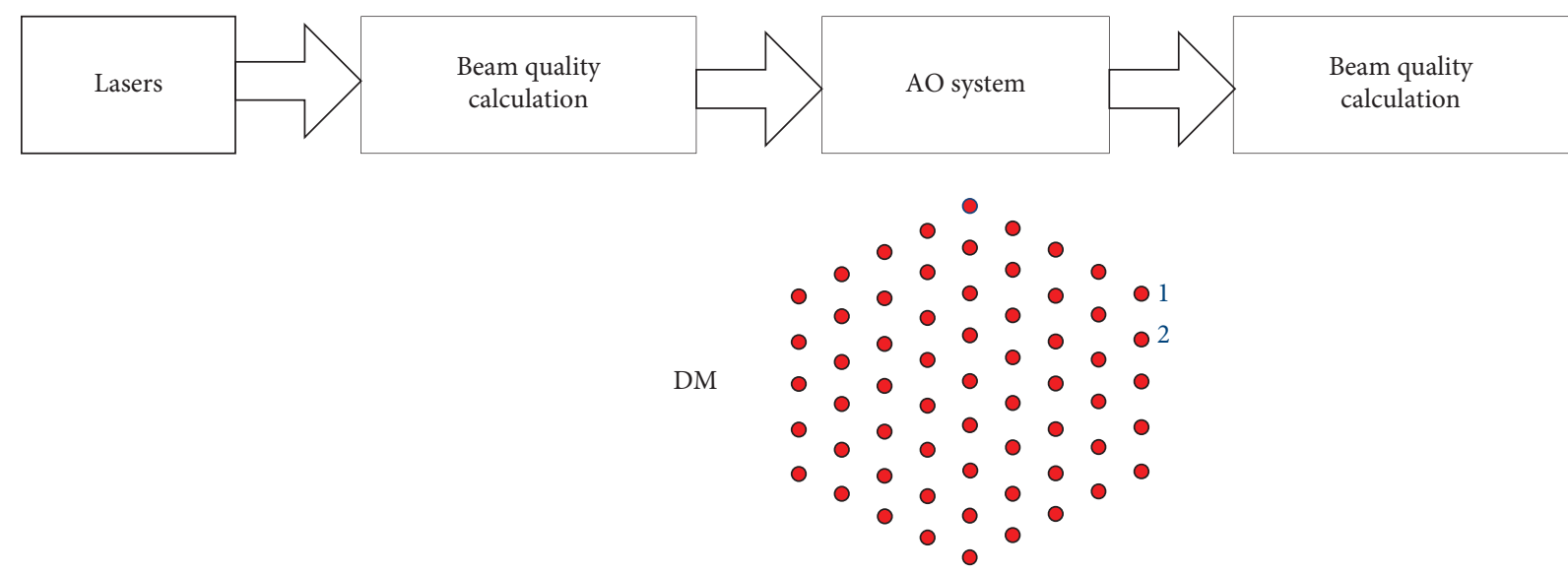

FIgURE 3: Schematic diagram of the model.

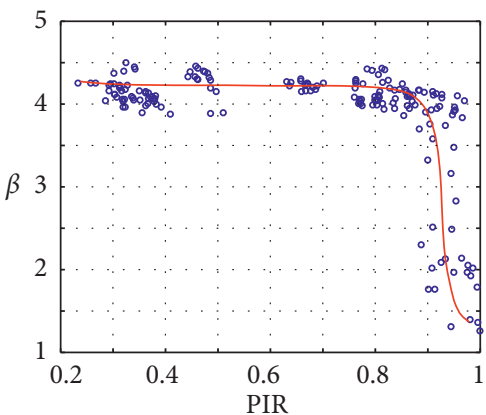

- Calculated data

- Fitting line

(a)

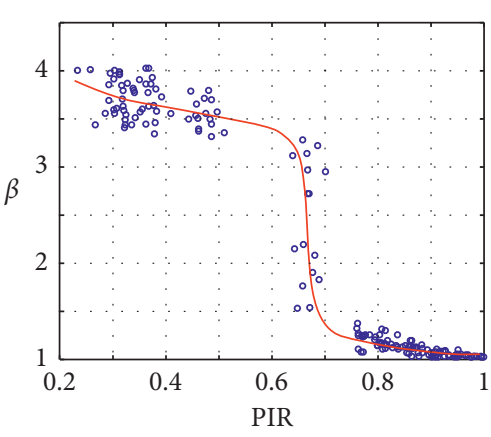

- Calculated data

- Fitting line

(d)

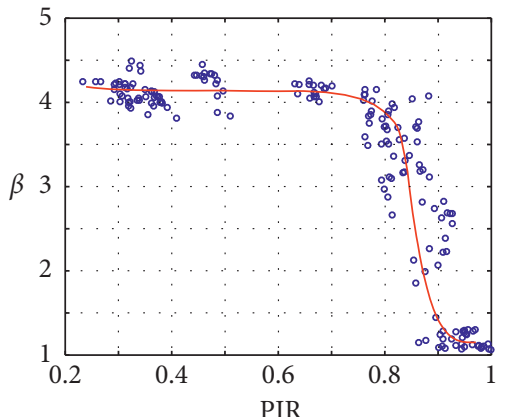

- Calculated data

_ Fitting line

(b)

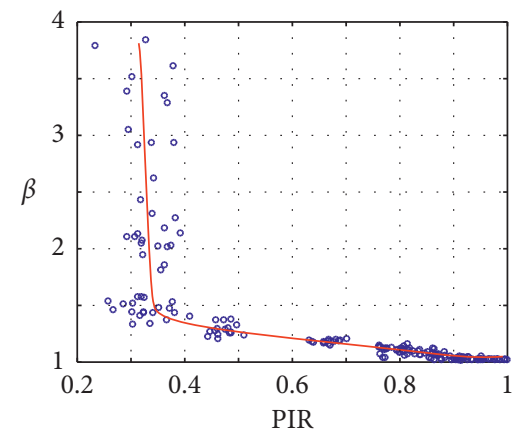

- Calculated data

- Fitting line

(e)

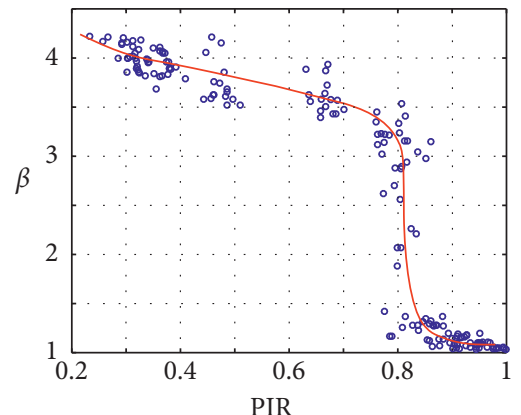

- Calculated data

__ Fitting line

(c)

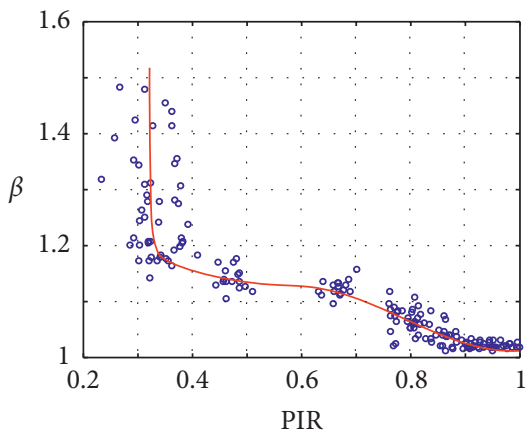

- Calculated data

_ Fitting line

(f)

FIGURE 4: Compensation effects of AO systems with different DMs: (a) 19 actuators, (b) 37 actuators, (c) 61 actuators, (d) 91 actuators, (e) 127 actuators, and (f) 169 actuators.

higher-order phase aberrations and the compensation effect gets better as well.

\section{Conclusions}

Based on the light intensity analysis of beams with different phase aberrations, a new beam quality evaluation factor PIR is presented in this paper. The PIR factor indicates low- and high-spatial frequency fractions in high-power laser beams, so it is able to evaluate the improvement potential by AO systems. Simulation results show that the closer the PIR values get to 1 , the closer the laser beams could be improved to the ideal. By detecting the compensation effects of beams with different PIR values and different DMs, it is well verified that the PIR could evaluate lasers' improvement potential using AO systems with different compensation capability. 


\section{Data Availability}

The simulation data used to support the findings of this study are available from the corresponding author upon request.

\section{Conflicts of Interest}

The authors declare that they have no conflicts of interest.

\section{References}

[1] G. Hunziker and C. Harder, "Beam quality of InGaAs ridge lasers at high output power," Applied Optics, vol. 34, no. 27, pp. 6118-6122, 1995.

[2] R. Hülsewede, J. Sebastian, H. Wenzel, G. Beister, A. Knauer, and G. Erbert, "Beam quality of high power $800 \mathrm{~nm}$ broadarea laser diodes with 1 and $2 \mu \mathrm{m}$ large optical cavity structures," Optics Communications, vol. 192, no. 1-2, pp. 69-75, 2001.

[3] M. Shayganmanesh and R. Beirami, "Evaluation of thermal effects on the beam quality of disk laser with unstable resonator," Optics Communications, vol. 383, pp. 92-100, 2017.

[4] J. D. Mansell, J. Hennawi, E. K. Gustafson et al., "Evaluating the effect of transmissive optic thermal lensing on laser beam quality with a Shack-Hartmann wave-front sensor," Applied Optics, vol. 40, no. 3, pp. 366-374, 2001.

[5] R. Lange and D. Kolbe, "Laser-controlled adaptive optics for beam quality improvements in a multi-pass thin-disk amplifier," Optics Letters, vol. 43, no. 14, pp. 3453-3456, 2018.

[6] E. Wyss, M. Roth, T. Graf, and H. P. Weber, "Thermooptical compensation methods for high-power lasers," IEEE Journal of Quantum Electronics, vol. 38, no. 12, pp. 1620-1628, 2002.

[7] Z. Ye, Y. Wang, Z. Zhao, C. Liu, and Z. Xiang, "Method to improve beam quality by compensating spherical aberrations in master oscillator power amplifier laser systems," Applied Optics, vol. 53, no. 33, pp. 7963-7967, 2014.

[8] M. A. Vorontsov and G. W. Carhart, "Adaptive phase distortion correction in strong speckle-modulation conditions," Optics Letters, vol. 27, no. 24, pp. 2155-2157, 2002.

[9] S. Jackel, I. Moshe, and R. Lavi, "Comparison of adaptive optics and phase-conjugate mirrors for correction of aberrations in double-pass amplifiers," Applied Optics, vol. 42, no. 6, pp. 983-989, 2003.

[10] B. Santanu and L. M. Gutheinz, "Fractional power in the bucket, beam quality and $M^{2}$," Proceedings of SPIE, vol. 7579, Article ID 75790U, 2010.

[11] B. Lü and H. Ma, "Coherent and incoherent combinations of off-axis Gaussian beams with rectangular symmetry," Optics Communications, vol. 171, no. 4-6, pp. 185-194, 1999.

[12] P. Yan, X. Wang, M. Gong, and Q. Xiao, "Evaluating the beam quality of double-cladding fiber lasers in applications," Applied Optics, vol. 55, no. 23, pp. 6145-6150, 2016.

[13] X. Fu, Q. Liu, P. Li, L. Huang, and M. Gong, "Numerical simulation of $30-\mathrm{kW}$ class liquid-cooled Nd:YAG multi-slab resonator," Optics Express, vol. 23, no. 14, pp. 18458-18470, 2015.

[14] X. Kang and B. Lü, "Vectorial nonparaxial flattened Gaussian beams and their beam quality in terms of the power in the bucket," Optics Communications, vol. 262, no. 1, pp. 1-7, 2006.

[15] A. Dixit, S. K. Mishra, and A. K. Gupta, "Sensitivity of singular beams in the presence of Zernike aberrations," Optics and Lasers in Engineering, vol. 71, pp. 74-84, 2015.
[16] H. Wang, H. Wang, X. Li, and S. Wang, "Intensity distribution for the broadband aberrated partially coherent flat-topped beam propagating through turbulent atmosphere," Optik-International Journal for Light and Electron Optics, vol. 124, no. 18, pp. 3554-3557, 2013.

[17] W. Jiang, "Relationship between beam quality factor $\beta$ and wave-front error," Chinese Journal of Lasers, vol. 32, no. 6 , pp. 799-802, 2005.

[18] Y. Qiu, L. Huang, M. Gong, L. Qiang, P. Yan, and H. Zhang, "Method to evaluate beam quality of Gaussian beams with aberrations," Applied Optics, vol. 51, no. 27, pp. 6539-6543, 2012.

[19] S. Chen, L. Dong, X. Chen et al., "Adaptive slab laser beam quality improvement using a weighted least-squares reconstruction algorithm," Applied Optics, vol. 55, no. 11, pp. 3077-3083, 2016.

[20] C. Mafusire and A. Forbes, "Generalized beam quality factor of aberrated truncated Gaussian laser beams," Journal of the Optical Society of America A, vol. 28, no. 7, pp. 1372-1378, 2011.

[21] M. Jiang, C. Zhou, W. Wang, P. Xi, and Q. Ren, "Comparative analysis of zernike aberrations generation with deformable mirrors for ocular adaptive optics," Journal of Modern Optics, vol. 56, no. 16, pp. 1741-1746, 2009.

[22] L. Zhu, P.-C. Sun, D.-U. Bartsch, W. R. Freeman, and Y. Fainman, "Wave-front generation of Zernike polynomial modes with a micromachined membrane deformable mirror," Applied Optics, vol. 38, no. 28, pp. 6019-6026, 1999. 

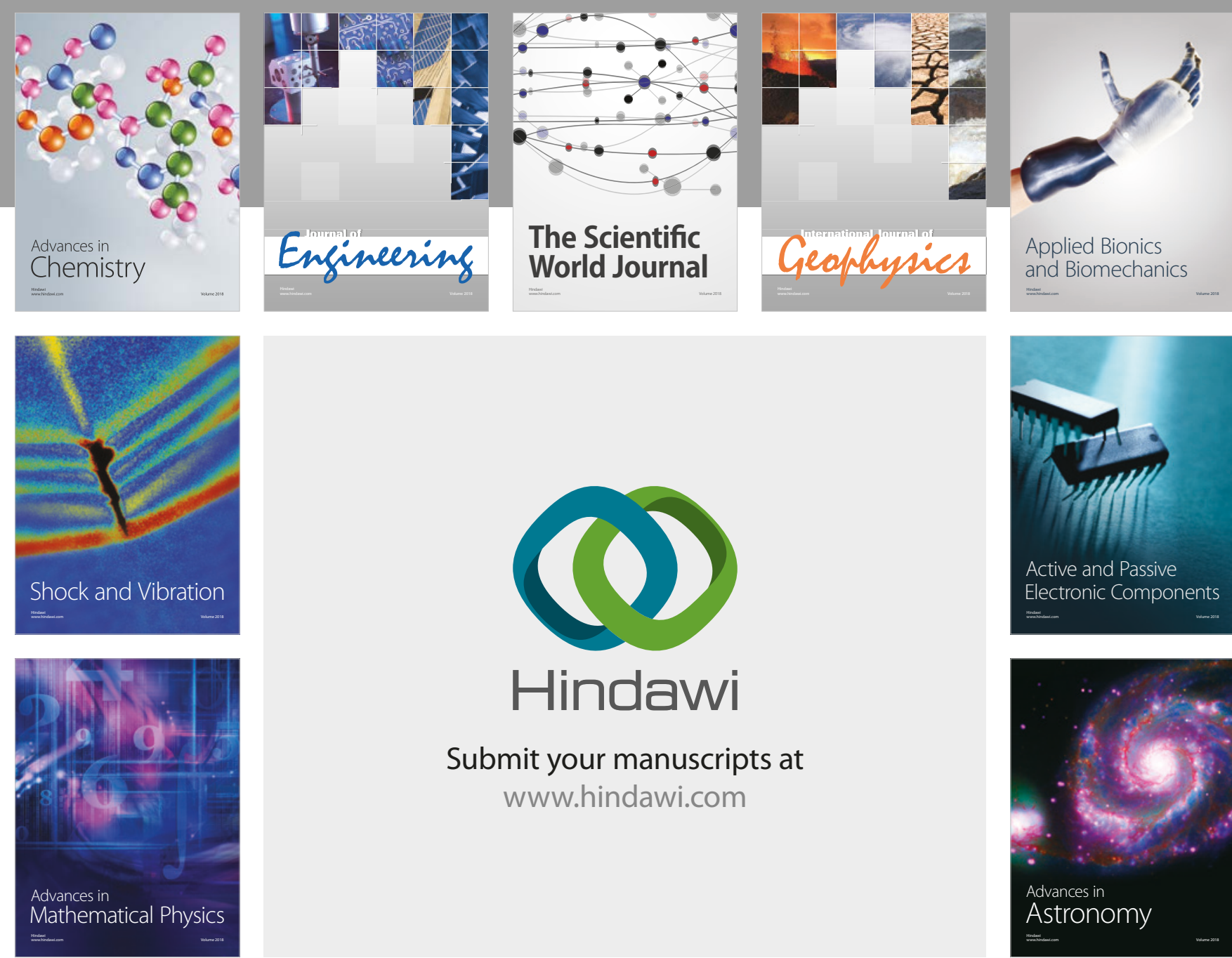

Submit your manuscripts at

www.hindawi.com

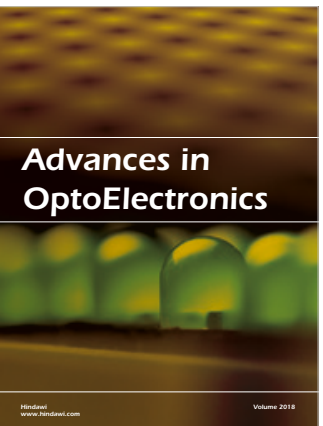

\section{Rotcting Machinery}
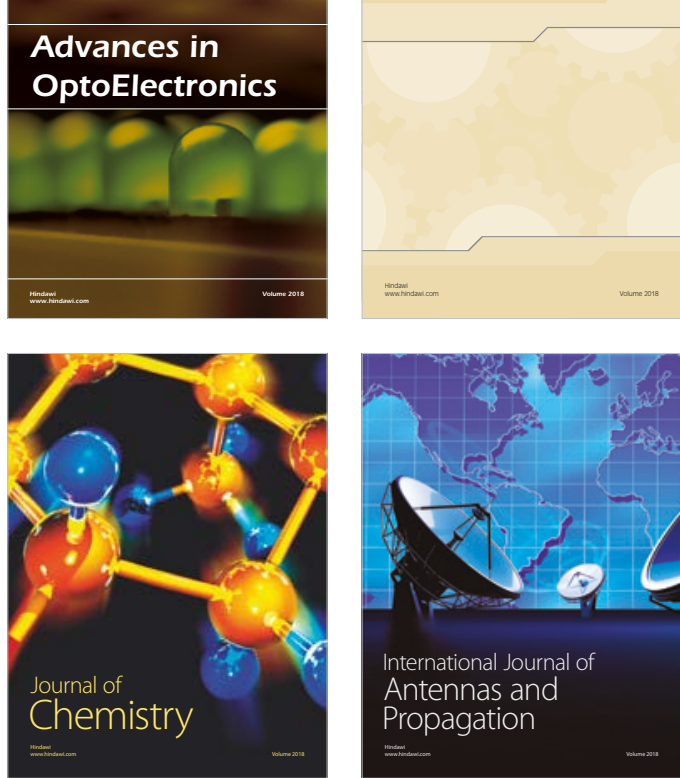

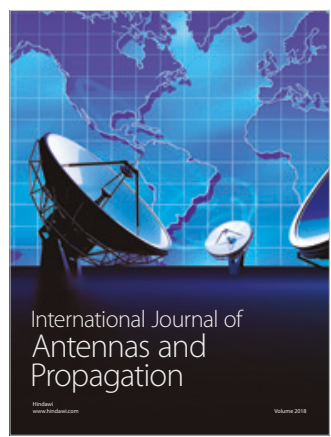

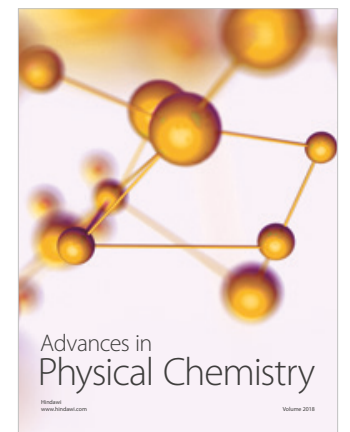

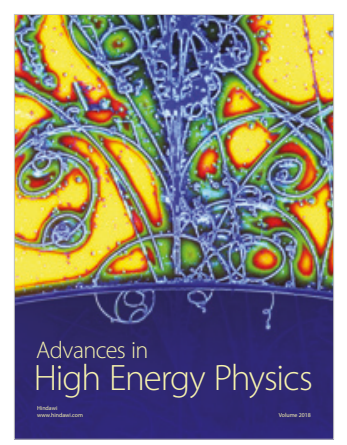

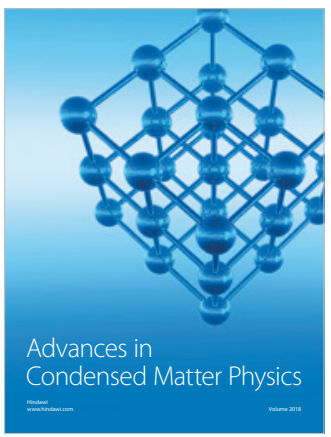

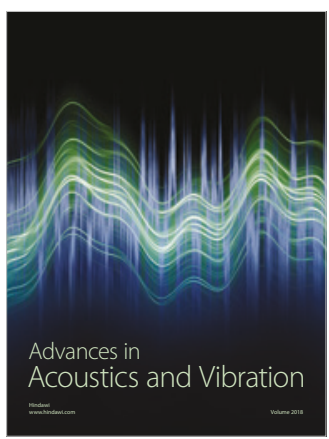

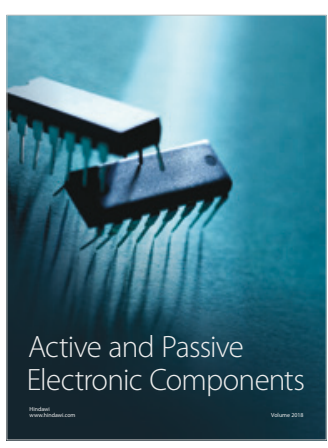
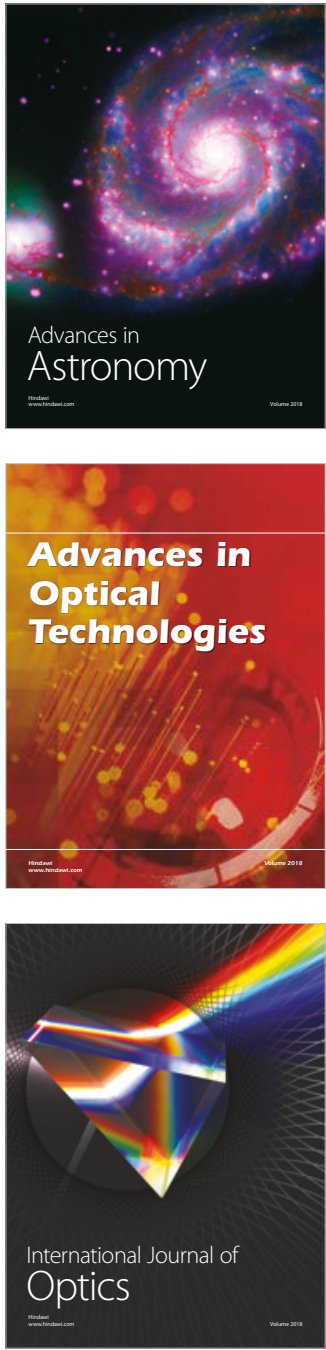